\title{
Study on Day and Night Sighting Device Target Imaging Simulation System
}

\author{
Lifeng Diao \\ First Aeronautical Institute of Air Force \\ Xinyang, China \\ aadlf@163.com \\ Cheng Yang \\ First Aeronautical Institute of Air Force \\ Xinyang, China \\ yrq9353@sohu.com
}

\author{
Ming Dai \\ First Aeronautical Institute of Air Force \\ Xinyang, China \\ xydming@126.com \\ Jilin $\mathrm{Wu}$ \\ First Aeronautical Institute of Air Force \\ Xinyang, China \\ flier_00783@sina.com
}

Guangdong Zha

First Aeronautical Institute of Air Force

Xinyang, China

dysx001@sohu.com

\begin{abstract}
Describes the design idea of an airborne day and night sighting device target imaging simulation system, the composition and working principle of simulation system is discussed from two aspects of hardware and software. The experimental result shows that the simulation system is with excellent image quality, can accurately calculate and display the tracking information.
\end{abstract}

Keywords- simulation; sensor; video capture

\section{INTRODUCTION}

An airborne day and night sighting device can acquire the television and infrared image of target on the ground, guide the laser guided bomb to attack the ground fixed and moving target by identifying and tracking the target image. Simulation system is designed according to the concept of imaging function of airborne day and night sighting device, simulate the generation, recognition and tracking of images, it is very important to familiar with the fight operation and upgrade the level of equipment maintenance training.

\section{THE COMPONENT OF IMAGING SIMULATION SYSTEM}

Day and night sighting device imaging simulation system is a data acquisition and control system as the core of digital computer, composed of hardware and software.

The hardware part consists of data acquisition subsystem, optical imaging subsystem, industrial control computer, motion control subsystem, motion platform and the power subsystem, the hardware block diagram shown in figure 1 .

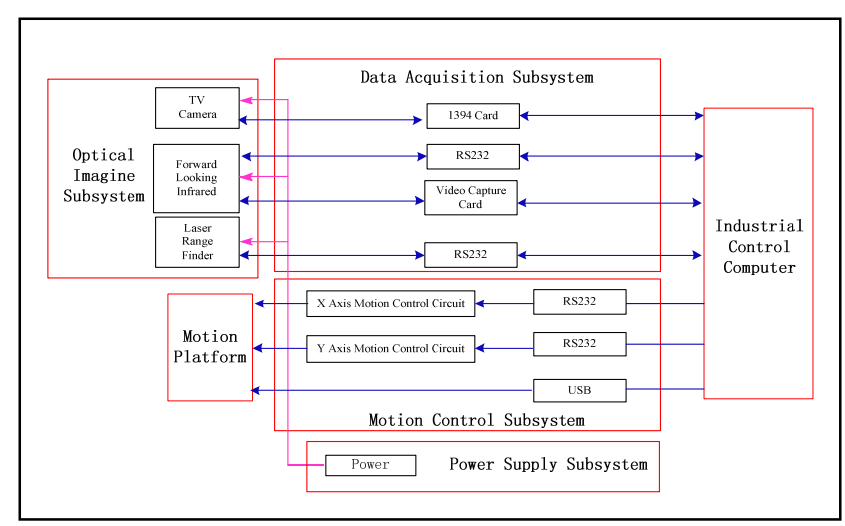

Figure 1. The hardware block diagram of Imaging Simulation System.

\section{A. Data Acquisition Subsystem}

Data acquisition subsystem is composed of 1394 cards, video capture card, RS232 etc.

The 1394 card is mainly used for color video signal acquisition of television cameras, and connected with industrial control computer by PCI bus, will acquire 1394 format video signal data into computer memory for the corresponding recognition, tracking processing through the acquisition of software.

Video capture card is mainly used for infrared camera gray signal acquisition, and is connected with industrial control computer by PCI bus, will acquire BNC format video signals data into computer memory for the corresponding recognition, tracking processing through the acquisition of software.

RS232 is mainly used for computer state control infrared camera and laser rangefinder ranging, using serial port resources of computer itself. 
When system is working, the optical imaging device is connected with industrial control computer through data acquisition subsystem. Under the control of acquisition software, video data is orderly mined into the corresponding data channel in certain protocols, the data is processed in computer memory, thus complete the identification, tracking function of imaging simulation system finally.

\section{B. Optical imaging subsystem}

Optical imaging subsystem is mainly used to achieve optical imaging and optical ranging of the television, infrared, is composed of television camera, infrared camera and laser range finder. Under the control of acquisition software, imaging data and distance data is acquired by data acquisition subsystem, and finally displayed on the terminal of LCD.

\section{Industrial control computer}

The host adopts industrial control computer. This machine is of high reliability, strong anti-interference ability, shockproof and moistureproof. All the information in the process of testing are displayed on the screen of computer. The industrial control computer is the control core of test equipment, and its bus structure provides the basis for the selection of mature hardware products. The method avoids duplication of development, also facilitates hardware upgrading.

\section{Motion control subsystem}

Motion control system is mainly used for the motion state control of motion platform, which is mainly composed of $\mathrm{X} / \mathrm{Y}$ rotation signal excitation circuit, pulse generating circuit, DC power supply circuit and motion parameter storage circuit.

$\mathrm{X} / \mathrm{Y}$ rotation signal excitation circuit is used for the generating of $\mathrm{X} / \mathrm{Y}$ motion signal, controls the movement of three directions.

The pulse generating circuit is used for the generating of motion signal excitation pulse, provides the $\mathrm{X} / \mathrm{Y}$ rotation signal excitation circuit.

DC power supply circuit generates 24V DC.

Motion parameter storage circuit stores the parameters of motion mode, angular velocity, pulse number etc.

\section{E. Motion platform}

Motion platform adopts shelf products, can realize the rotation of two degrees of freedom and ensure stable motion of imaging equipment in the control of motion systems.

\section{F. Power supply subsystem}

Power supply subsystem provides special power to the component of Imaging Simulation System.

\section{SOFTWARE DESIGN FOR IMAGING SIMULATION SYSTEM}

In order to fulfill the requirement of universal and extensible, the software of imaging simulation system adopts standard communication protocol and common development environment, thus the system can achieve the parathion of hardware and software. In order to guarantee the system's interference, software adopts the separation technology of the acquisition equipment and acquisition object. Software is mainly composed of initialization program, acquisition program, recognition tracking model, motion control parameters configuration files, help document. Software components as shown in figure 2 .

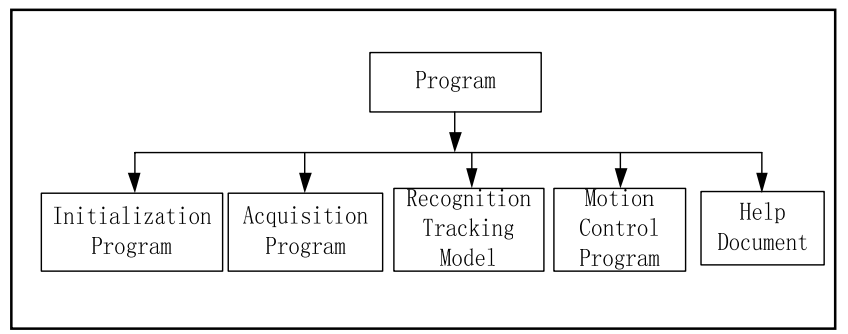

Figure 2. Software components of imaging simulation system.

The initialization program completes self-detection while the system is power on.

Acquisition program is the core of imaging simulation system, responsible for the definition of different protocol data stream, capture model management, acquisition flow control, online help etc.

Here, acquisition program itself does not contain any hardware information of imaging device, is completely independent. It can realize the management and driving of acquisition hardware by the initialization procedure. If the acquisition hardware changes, just change the initialization program. Acquisition program realizes the image acquisition process control by acquisition model. The acquisition model changes if the acquisition hardware changes.

Recognition tracking model is based on image processing technique, built by classifying television, infrared target shape, which consists of a Microsoft SQL database, display text and report text.

Motion control program is the basis of target tracking, responsible for motion parameter setting and motion state control of the motion platform.

Help document is to display help.

\section{A. Initialization program}

The initialization program contains information about the acquisition hardware, the deriving, inheriting relationship of hardware management object as shown in figure 3: 


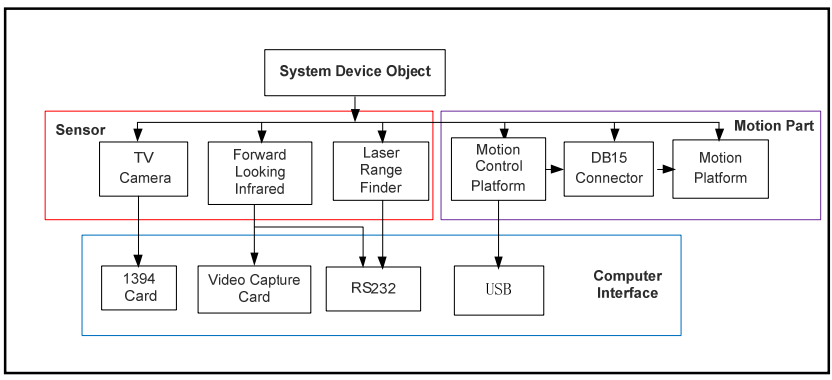

Figure 3. The deriving, inheriting relationship of hardware management object.

\section{B. Acquisition program}

Acquisition program adopts object-oriented (OO) technology, is composed of three parts: analysis (OOA), design (OOD) and programming (OOP). It mainly includes the following subjects: total control object, data stream object, acquisition model management object and help object.

Total control object is the control center of imaging simulation system for the main interface displaying and user operation scheduling.

Data stream object is for the definition of different types of data protocol.

Acquisition model management object is used to establish, edit, delete and interpret acquisition model. It provides design wizard of acquisition model. With the wizard user does not need to know the detailed structure of acquisition model, can design complex acquisition model by simple selecting operation. The method can reduce the difficulty of development and greatly improve the speed of development. At the same time, acquisition program interprets the acquisition model by calling the acquisition model object, controls the acquisition flow and provides the operation tips, to realize the automatic acquisition of the data.

Help object is used to provide detailed help.

\section{Recognition tracking model}

Recognition tracking model contains the key features of the imaging target, is composed of Microsoft SQL database, display text and report text.

The database is a hierarchical system, mainly composed of primitive image data, gray image processing data and image contour data.

The display text includes prompt information when system chooses tracking algorithm (correlation, contrast).

The report text is used to generate document template of distance, tracking azimuth and elevation angle results.

These text files are stored in a linear manner, referred in a nonlinear manner.

\section{Motion control program}

Motion control program stores the hardware information of motion console, such as device number, USB port, and state parameters of the motion control subsystem, including the mode of motion, angular velocity and pulse number etc.
The precision of motion control program influence television, infrared imaging target searching and tracking precision. According to the target motion characteristics, rules and tracking algorithm, motion control program can control the motion platform to pitch, roll in accurate direction by calculate the correct tracking azimuth and elevation angle.

\section{E. Help document}

Help document including the software requirements specification, data requirements specifications, acquisition technical qualification data, program design specifications, database design specification, user manuals etc. Some of these documents are used for software development, upgrade and maintenance, some are provided to the user. These documents ensure the standardization and engineer characteristic of software development.

The basic working process of the day and night sighting device target imaging simulation system is that at first fulfill the device hardware information matching and selfinspection by initialization program, so as to determine whether the test equipment itself is in good condition. When self-inspection is completed, the system runs the program for collecting video, to obtain stable video image, then set the day and night sighting device into the servo state manually. At this time, identification tracking module start running automatically to enter the tracking state, the software will display the tracking status information or generate tracking results reports and print to output according to the user requirements.

Visual display and convenient use are the main characteristics of this simulation software. It can set the type of equipment automatically and select the corresponding imaging function, including: unlock / lock, TV / IR switching, black / white heat switching, wide / narrow field of view switching, ranging, tracking and touch control etc.

\section{CONCLUSION}

The simulation system can be used for research on television, infrared images processing and verification of target recognition tracking problems base on digital image processing technology and the motion control technology. It can be operated according to the function of true airborne day and night sighting device, is great significance to improve the maintenance and training of equipment.

\section{REFERENCES}

[1] TIAN Xiaowei, "Research on tracking algorithms of moving objects" Changchun: Changchun University of Science and Techonlogy, 2008.

[2] LIU Songtao, ZHOU Xiaodong, SHEN Tongsheng, "The algorithm verification system of target recognition and tracking using $\mathrm{VC}++$ 6.0”, Computer Applications and Software, 2004,vol 21, pp.80-81.

[3] YANG Geng, WEI Xing, "Study of system on image recognition and tracking of moving object," Computer Measurement and Control, 2005,vol 13 , pp. 267-269.

[4] MAO Zheng, SUN Legong,WU Liang, "Design anbbgnx cv cxfcdfsd realization of air2to2ground TV2guided weapon simulation system" System Simulation Technology, 2010, vol 6 , pp. 140-146. 